\title{
Equipment of sets with cardinality of the continuum by structures of Polish groups with Haar measures
}

\author{
Gogi Rauli Pantsulaia \\ Department of mathematics Georgian Technical University \\ Tbilisi , Republic of Georgia \\ g.pantsulaia@gtu.ge
}

Keywords: Polish space; Polish group; Lie group; Haar measure; two-sided invariant measure

\begin{abstract}
It is introduced a certain approach for equipment of sets with cardinality of the continuum by structures of Polish groups with two-sided (left or right) invariant Haar measures. By using this approach we answer positively Maleki's certain question(2012) what are the real k-dimensional manifolds with at least two different Lie group structures that have the same Haar measure. It is demonstrated that for each diffused Borel probability measure $\mu$ defined in a Polish space $\left(G, \rho, \mathcal{B}_{\rho}(G)\right)$ without isolated points there exist a metric $\rho_{1}$ and a group operation $\odot$ in $G$ such that $\mathcal{B}_{\rho}(G)=\mathcal{B}_{\rho_{1}}(G)$ and $\left(G, \rho_{1}, \mathcal{B}_{\rho_{1}}(G), \odot\right)$ stands a compact Polish group with a two-sided (left or right) invariant Haar measure $\mu$, where $\mathcal{B}_{\rho}(G)$ and $\mathcal{B}_{\rho_{1}}(G)$ denote Borel $\sigma$-algebras of subsets of $G$ generated by metrics $\rho$ and $\rho_{1}$, respectively. Similar approach is used for a construction of locally compact non-compact or non-locally compact Polish groups equipped with two-sided (left or right) invariant quasi-finite Borel measures.
\end{abstract}

\section{Introduction}

Let $(G, \rho, \odot)$ be a Polish group, by which we mean a group with a complete separable metric $\rho$ for which the transformation (from $G \times G$ onto $G$ ) sending $(x, y)$ into $x^{-1} \odot y$ is continuous.

We denote by $B_{\rho}(G)$ the $\sigma$-algebra of Borel subsets of $G$ defined by the metric $\rho$.

The following problem was under intensive consideration by many mathematicians exactly one century ago.

Problem 1.1. Let $(G, \rho, \odot)$ be a locally compact Polish group which is dense-in-itself ${ }^{1}$, that is, a space homeomorphic to a separable complete metric space and $G$ has no isolated points. Does there exist a Borel measure $\mu$ in $(G, \rho, \odot)$ satisfying the following properties:

(i) The measure $\mu$ is diffused, that is, $\mu$ vanishes on all singletons;

(ii) The measure $\mu$ is a two-sided (left or right) invariant, that is, $\mu\left(g_{1} \odot E \odot g_{2}\right)=\mu(E)\left(\mu\left(g_{1} \odot\right.\right.$ $E)=\mu(E)$ or $\left.\mu\left(E \odot g_{2}\right)=\mu(E)\right)$ for every $g_{1}, g_{2} \in G$ and every Borel set $E \in B(G)$;

(iii) The measure $\mu$ is outer regular, that is,

$$
(\forall E)(E \in B(G) \rightarrow \mu(E)=\inf \{\mu(U): E \subseteq U \& U \text { is open }\}) ;
$$

(iv) The measure $\mu$ is inner regular, that is,

$$
(\forall E)(E \in B(G) \rightarrow \mu(E)=\sup \{\mu(F): F \subseteq E \& F \text { is compact }\}) ;
$$

(v) The measure $\mu$ is finite on every compact set, that is $\mu(K)<\infty$ for all compact $K$.

The special case of a left (or right ) invariant measure for second countable ${ }^{2}$ locally compact groups had been shown by Haar in 1933 [8]. Note that each Polish space is second countable which

\footnotetext{
${ }^{1}$ A subset $A$ of a topological space is said to be dense-in-itself if $A$ contains no isolated points.

${ }^{2}$ A topological space $T$ is second countable if there exists some countable collection $\mathcal{U}=\left\{U_{i}\right\}_{i \in N}$ of open subsets of $T$ such that any open subset of $T$ can be written as a union of elements of some subfamily of $\mathcal{U}$
} 
implies that the answer to Problem 1.1 is yes. The measure $\mu$ satisfying conditions (i)-(v) is called a left (right or two-sided) invariant Haar measure in a locally compact Polish group $(G, \rho, \odot)$.

In this note we would like to study the following problems, which can be considered as converse (in some sense) to Problem 1.1.

Problem 1.2. Let $(G, \rho)$ be a Polish metric space which is dense-in-itself. Let $\mu$ be a diffused Borel probability measure defined in $(G, \rho)$. Do there exist a metric $\rho_{1}$ and a group operation $\odot$ in $G$ such that the following three conditions

(j) The class of Borel measurable subsets of $G$ generated by the metric $\rho_{1}$ coincides with the class of Borel measurable subsets of the same space generated by the metric $\rho$,

(jj) $\left(G, \rho_{1}, \odot\right)$ is a compact Polish group

and

(jij) $\mu$ is a left(right or two-sided) invariant Haar measure in $\left(G, \rho_{1}, \odot\right)$

hold true?

For a solution of the Problem 1.2 , there will be used especially an existence of Borel isomorphism between two diffused Borel probability measures in Polish groups (cf. [3]).

Problem 1.3. Let $(G, \rho)$ be a Polish metric space which is dense-in-itself. Let $\mu$ be a diffused $\sigma$-finite non-finite Borel measure defined in $(G, \rho)$. Do there exist a metric $\rho_{\varphi}$, a group operation $\odot_{\varphi}$ in $G$ and the Borel measure $\mu^{\star}$ in $G$ such that the following four conditions

(i) The class of Borel measurable subsets of $G$ generated by the metric $\rho_{\varphi}$ coincides with the class of Borel measurable subsets of the same space generated by the metric $\rho$,

(ii) $\left(G, \rho_{\varphi}, \odot_{\varphi}\right)$ is a non-compact locally compact Polish group,

(iii) The measures $\mu^{\star}$ and $\mu$ are equivalent

and

(iv) $\mu^{\star}$ is a left (right or two-sided) invariant $\sigma$-finite non-finite Haar measure in $\left(G, \rho_{\varphi}, \odot_{\varphi}\right)$ hold true?

Problem 1.4. Let $(G, \rho)$ be a Polish metric space which is dense-in-itself. Let $\mu$ be a diffused non- $\sigma$ quasi-finite Borel measure defined in $(G, \rho)$. Do there exist a metric $\rho_{1}$ and a group operation $\odot$ in $G$ such that the following three conditions

(j) The class of Borel measurable subsets of $G$ generated by the metric $\rho_{1}$ coincides with the class of Borel measurable subsets of the same space generated by the metric $\rho$,

(jj) $\left(G, \rho_{1}, \odot\right)$ is a non-locally compact Polish group

and

(jjj) $\mu$ is a left(right or two-sided) invariant quasi-finite Borel measure in $\left(G, \rho_{1}, \odot\right)$

hold true?

In [4], the author uses methods of the theory ultrafilters to present a modified proof that a locally compact group with a countable basis has a left invariant and right invariant Haar measure. The author first shows that the topological space $\left(\beta_{1} X ; \tau_{1}\right)$ consisting of all ultrafilters on a non-empty set $X$ is homeomorphic to the topological space $\left(\beta_{2} X ; \tau_{2}\right)$ of all nonzero multiplicative functions in the first dual space $\ell_{\infty}^{*}(X)$ (Theorem 3.8). By using this result the author proves the existence of the infinitely additive left invariant measure $\lambda$ on compact sets of the locally compact Hausdorf topological group $G$ (Theorem 7.1). Starting from this point, the author introduces the notion of $\nu$-measurable subsets in $G$ where $\nu$ is an outer measure in $G$ induced by the $\lambda$ and open sets in $G$, and proves the existence of a left invariant Haar measure by the scheme presented in [7]. Notice that his proof essentially uses the axiom of choice. Several examples of the Haar measure are presented. It is underlined by Example 9.7 that $G=R^{k}$ with $k=\frac{n^{2}-n}{2}$ has two Lie group structures but the Lebesgue measure on $R^{k}$ is the Haar measure on both Lie groups. In this context the following question was stated in this paper. 
Problem 1.5([4],Question 9.8) What are the real $k$-dimensional manifolds with at least two different Lie group structures that have the same Haar measure?

The rest of the present manuscript is the following.

In Section 2 we introduce a certain approach for an equipment of an arbitrary set of the cardinality of the continuum by structures of various(compact, locally compact or non-locally compact) Polish groups with two-sided(left or right) invariant Borel measures. Problems 1.2-1.5 will be stutied also in this section.

In Section 3 we consider a question asking whether an arbitrary diffused Borel probability measure in a Polish space without isolated points can be considered as a Haar measure.

\section{Equipment of an arbitrary set with cardinality of the continuum with structures of Polish groups and Haar's measures}

This section we begin by description of a certain useful approach which allows us to equip sets with cardinality of the continuum with structures of Polish groups and Haar's measures. Since next theorem (as we will see later) has many interesting applications, we give its proof in detail.

Theorem 2.1 Let $X$ be a set with cardinality of the continuum and $(G, \odot, \rho)$ be a Polish group. Further, let $f: G \rightarrow X$ be a one-to-one mapping. We set

$$
x \odot_{f} y=f\left(f^{-1}(x) \odot f^{-1}(y)\right)
$$

and

$$
\rho_{f}(x, y)=\rho\left(f^{-1}(x), f^{-1}(y)\right)
$$

for $x, y \in X$. Then the following conditions hold true:

(i) $\left(G_{f}, \odot_{f}, \rho_{f}\right)$ is a Polish group which is Borel isomorphic to the Polish group $(G, \odot, \rho)$;

(ii) If $(G, \odot, \rho)$ is an abelian Polish group then so is $\left(G_{f}, \odot_{f}, \rho_{f}\right)$;

(iii) If $\rho$ is two-sided invariant metric in $(G, \odot)$ so is $\rho_{f}$ in $\left(G_{f}, \odot_{f}\right)$;

(iv) If $(G, \odot, \rho)$ is dense-in-itself so is $\left(X, \odot_{f}, \rho_{f}\right)$;

(v) If $(G, \odot, \rho)$ is a compact Polish group then so is $\left(X, \odot_{f}, \rho_{f}\right)$;

(vi) If $(G, \odot, \rho)$ is a locally compact Polish group then so is $\left(X, \odot_{f}, \rho_{f}\right)$;

(vii) If $(G, \odot, \rho)$ a non-locally compact Polish group then so is $\left(X, \odot_{f}, \rho_{f}\right)$;

(viii) If $(G, \odot, \rho)$ is a locally compact or compact Polish group and $\lambda$ is a left(or right or twosided ) invariant Haar measure in $(G, \odot, \rho)$, then $\lambda_{f}$ also is a left(or right or two-sided ) invariant Haar measure in $\left(G_{f}, \odot_{f}, \rho_{f}\right)$, where $G_{f}=X, \mathcal{B}_{\rho_{f}}\left(G_{f}\right)$ is Borel $\sigma$-algebra of $G_{f}$ generated by the metric $\rho_{f}$ and $\lambda_{f}$ is a Borel measure in $G_{f}$ defined by

$$
(\forall Y)\left(Y \in \mathcal{B}_{f}\left(G_{f}\right) \rightarrow \lambda_{f}(Y)=\lambda\left(f^{-1}(Y)\right)\right) .
$$

(ix) If $(G, \odot, \rho)$ is a non-locally compact Polish group and $\lambda$ is a left(or right or two-sided) invariant quasi-finite ${ }^{3}$ Borel measure in $(G, \odot, \rho)$, then $\lambda_{f}$ also is a left(or right or two-sided) invariant quasi-finite Borel measure in $\left(G_{f}, \odot_{f}, \rho_{f}\right)$, where $G_{f}=X, \mathcal{B}_{\rho_{f}}\left(G_{f}\right)$ is Borel $\sigma$-algebra of $G_{f}$ generated by the metric $\rho_{f}$ and $\lambda_{f}$ is a Borel measure in $G_{f}$ defined by

$$
(\forall Y)\left(Y \in \mathcal{B}_{f}\left(G_{f}\right) \rightarrow \lambda_{f}(Y)=\lambda\left(f^{-1}(Y)\right)\right) .
$$

Proof. Proof of the item (i).

Closure. If $x, y \in X$ then $x \odot_{f} y=f\left(f^{-1}(x) \odot f^{-1}(y)\right) \in X$.

Associativity. For all $x, y$ and $z$ in $X$, we have

\footnotetext{
${ }^{3} \mathrm{~A}$ measure $\mu$ is called quasi-finite if there is a $\mu$-measurable set $X$ with $0<\mu(X)<+\infty$.
} 


$$
\begin{gathered}
\left(x \odot_{f} y\right) \odot_{f} z=f\left[f^{-1}\left(x \odot_{f} y\right) \odot f^{-1}(z)\right]=f\left[f^{-1}\left(f\left(f^{-1}(x) \odot f^{-1}(y)\right)\right) \odot f^{-1}(z)\right]= \\
f\left[\left(f^{-1}(x) \odot f^{-1}(y)\right) \odot f^{-1}(z)\right]=f\left[f^{-1}(x) \odot\left(f^{-1}(y) \odot f^{-1}(z)\right)\right]= \\
f\left[f^{-1}(x) \odot f^{-1}\left(y \odot_{f} z\right)\right]=x \odot_{f}\left(y \odot_{f} z\right) .
\end{gathered}
$$

Identity element. Let $e$ be an identity element of $G$. Setting $e_{f}:=f(e) \in X$, for $x \in X$ we have

$$
x \odot_{f} e_{f}=x \odot_{f} f(e)=f\left(f^{-1}(x) \odot f^{-1}(f(e))\right)=f\left(f^{-1}(x) \odot e\right)=f\left(f^{-1}(x)\right)=x
$$

and

$$
e_{f} \odot_{f} x=f(e) \odot_{f} x=f\left(f^{-1}(f(e)) \odot f^{-1}(x)\right)=f\left(e \odot f^{-1}(x)\right)=f\left(f^{-1}(x)\right)=x
$$

The latter relations means that $e_{f}$ is the identity element of $X$.

Inverse element. If $a \in G$ then we denote its inverse element by $a_{G}^{-1}$. For $x \in X$ setting $x_{X}^{-1}=$ $f\left(\left(f^{-1}(x)\right)_{G}^{-1}\right)$, we have

$$
\begin{gathered}
x \odot_{f} x_{X}^{-1}=f\left(f^{-1}(x) \odot f^{-1}\left(x_{X}^{-1}\right)\right)=f\left(f^{-1}(x) \odot f^{-1}\left(f\left(\left(f^{-1}(x)\right)_{G}^{-1}\right)\right)\right)= \\
f\left(f^{-1}(x) \odot\left(f^{-1}(x)\right)_{G}^{-1}\right)=f(e)=e_{f}
\end{gathered}
$$

and

$$
\begin{gathered}
x_{X}^{-1} \odot_{f} x=f\left(f^{-1}\left(x_{X}^{-1}\right) \odot f^{-1}(x)\right)=f\left(f^{-1}\left(f\left(\left(f^{-1}(x)\right)_{G}^{-1}\right)\right) \odot f^{-1}(x)\right)= \\
f\left(\left(f^{-1}(x)\right)_{G}^{-1} \odot f^{-1}(x)\right)=f(e)=e_{f} .
\end{gathered}
$$

The latter relations means that $x_{X}^{-1}$ is an inverse element of $x$.

Continuity of the operation $(x, y) \rightarrow x \odot_{f} y_{X}^{-1}$ when $(a, b) \rightarrow a \odot b_{G}^{-1}$ is continuous.

For all neighbourhood $U_{X}\left(x \odot_{f} y_{X}^{-1}, r\right)$ we have to choose such neighbourhoods $U_{X}\left(x, r_{1}\right)$ and $U_{X}\left(y, r_{2}\right)$ of elements $x$ and $y$ respectively that $\left(w_{1} \odot_{f}\left(w_{2}\right)_{X}^{-1}\right) \in U_{X}\left(x \odot_{f} y_{X}^{-1}, r\right)$ for $w_{1} \in U_{X}\left(x, r_{1}\right)$ and $w_{2} \in U_{X}\left(y, r_{2}\right)$.

We have

$$
\begin{gathered}
U_{X}\left(x \odot_{f} y_{X}^{-1}, r\right)=\left\{z: \rho_{f}\left(z, x \odot_{f} y_{X}^{-1}\right)<r\right\}=\left\{z: \rho\left(f^{-1}(z), f^{-1}\left(x \odot_{f} y_{X}^{-1}\right)\right)<r\right\}= \\
\left\{z: \rho\left(f^{-1}(z), f^{-1}\left(f\left(f^{-1}(x) \odot f^{-1}\left(y_{X}^{-1}\right)\right)\right)\right)<r\right\}= \\
\left\{z: \rho\left(f^{-1}(z), f^{-1}\left(f\left(f^{-1}(x) \odot f^{-1}\left(f\left(\left(f^{-1}(y)\right)_{G}^{-1}\right)\right)\right)\right)<r\right\}=\right. \\
\left.\left.\left\{z: \rho\left(f^{-1}(z),\left(f^{-1}(x) \odot\left(f^{-1}(y)\right)_{G}^{-1}\right)\right)\right)\right)<r\right\} .
\end{gathered}
$$

Since $(a, b) \rightarrow a \odot b_{G}^{-1}$ is continuous, for $a=f^{-1}(x), b=f^{-1}(y)$ and $r>0$ we can choose such neighbourhoods $U_{G}\left(f^{-1}(x), r_{1}\right)$ and $U_{G}\left(f^{-1}(y), r_{2}\right)$ of elements $f^{-1}(x)$ and $f^{-1}(y)$ respectively that then $\left(a_{1} \odot\left(a_{2}\right)_{G}^{-1}\right) \in U_{G}\left(f^{-1}(x) \odot\left(f^{-1}(y)\right)_{G}^{-1}, r\right)$ for $a_{1} \in U_{G}\left(f^{-1}(x), r_{1}\right)$ and $a_{2} \in U_{G}\left(f^{-1}(y), r_{2}\right)$.

It is obvious to check the validity of the following equalities

$$
\begin{gathered}
U_{X}\left(x, r_{1}\right)=f\left(U_{G}\left(f^{-1}(x), r_{1}\right)\right), \\
U_{X}\left(y, r_{2}\right)=f\left(U_{G}\left(f^{-1}(y), r_{2}\right)\right), \\
U_{X}\left(x \odot_{f} y_{X}^{-1}, r\right)=f\left(U_{G}\left(f^{-1}(x) \odot\left(f^{-1}(y)\right)_{G}^{-1}, r\right) .\right.
\end{gathered}
$$

Notice that if $w_{1} \in U_{X}\left(x, r_{1}\right)$ and $w_{2} \in U_{X}\left(y, r_{2}\right)$ then $\left(w_{1} \odot_{f}\left(w_{2}\right)_{X}^{-1}\right) \in U_{X}\left(x \odot_{f} y_{X}^{-1}, r\right)$. Indeed, $w_{1} \in U_{X}\left(x, r_{1}\right)$ and $w_{2} \in U_{X}\left(y, r_{2}\right)$ imply that $f^{-1}\left(w_{1}\right) \in U_{G}\left(f^{-1}(x), r_{1}\right)$ and $f^{-1}\left(w_{2}\right) \in$ $U_{G}\left(f^{-1}(y), r_{2}\right)$ from which we deduce that $\left(w_{1} \odot\left(w_{2}\right)_{G}^{-1}\right) \in U_{G}\left(f^{-1}(x) \odot\left(f^{-1}(y)\right)_{G}^{-1}, r\right)$. 
Borel isomorphism of $(G, \odot, \rho)$ and $\left(G_{f}, \odot_{f}, \rho_{f}\right)$. Notice that this isomorphism is realized by the mapping $f: G \rightarrow G_{f}$.

Proof of the item (ii).

Since $(G, \odot)$ is an abelian Polish group, for $x, y \in G_{f}$ we have

$$
x \odot_{f} y=f\left(f^{-1}(x) \odot f^{-1}(y)\right)=f\left(f^{-1}(y) \odot f^{-1}(x)\right)=y \odot_{f} x .
$$

Proof of the item (iii).

Since $\rho$ is a two-sided invariant metric in $(G, \odot)$ we have $\rho\left(h_{1} \odot x \odot h_{2}, h_{1} \odot y \odot h_{2}\right)=\rho(x, y)$ for each $x, y, h_{1}, h_{2} \in G$. Take into account this fact and the associativity property of the group operation $\odot_{f}$, we get that the condition

$$
\begin{gathered}
\rho_{f}\left(h_{1}^{*} \odot_{f} x^{*} \odot_{f} h_{2}^{*}, h_{1}^{*} \odot_{f} y^{*} \odot_{f} h_{2}^{*}\right)= \\
\rho_{f}\left(f\left(f^{-1}\left(h_{1}^{*}\right) \odot f^{-1}\left(x^{*}\right) \odot f^{-1}\left(h_{2}^{*}\right)\right), f\left(f^{-1}\left(h_{1}^{*}\right) \odot f^{-1}\left(y^{*}\right) \odot f^{-1}\left(h_{2}^{*}\right)\right)\right)= \\
\rho\left(f^{-1}\left(h_{1}^{*}\right) \odot f^{-1}\left(x^{*}\right) \odot f^{-1}\left(h_{2}^{*}\right), f^{-1}\left(h_{1}^{*}\right) \odot f^{-1}\left(y^{*}\right) \odot f^{-1}\left(h_{2}^{*}\right)\right)= \\
\rho\left(f^{-1}\left(x^{*}\right), f^{-1}\left(y^{*}\right)\right)=\rho_{f}\left(f\left(f^{-1}\left(x^{*}\right)\right), f\left(f^{-1}\left(y^{*}\right)\right)\right)=\rho_{f}\left(x^{*}, y^{*}\right)
\end{gathered}
$$

holds true for each $x^{*}, y^{*}, h_{1}^{*}, h_{2}^{*} \in G_{f}$.

Proof of the item (iv). We have to show that if $(G, \odot, \rho)$ is dense-in-itself then so is $\left(G_{f}, \odot_{f}, \rho_{f}\right)$. Indeed assume the contrary and let $x^{*}$ be an isolated point of $G_{f}$. The latter relation means that for some $\epsilon>0$ we have $\rho_{f}\left(y^{*}, x^{*}\right) \geq \epsilon$ for each $y^{*} \in G_{f} \backslash\left\{x^{*}\right\}$ which implies that $\rho(y, x) \geq \epsilon$ for each $y \in G \backslash\{x\}$ where $x=f^{-1}\left(x^{*}\right)$. We get the contradiction and the validity of the item (iv) is proved.

Proof of the item (v). We have to prove that if a family of open sets $\left(U_{i}^{*}\right)_{i \in I}$ whose union covers the space $G_{f}$ then there is its subfamily whose union also covers the same space. Let consider a family of sets $\left(f^{-1}\left(U_{i}^{*}\right)\right)_{i \in I}$. Since it is the family of open sets whose union covers the space $G$ and $G$ is a compact space, we claim that there is a finite subfamily $\left(f^{-1}\left(U_{i_{k}}^{*}\right)\right)_{1 \leq k \leq n}\left(i_{k} \in I\right.$ for $\left.k=1, \cdots, n\right)$ ) whose union $\cup_{k=1}^{n} f^{-1}\left(U_{i_{k}}^{*}\right)$ covers $G$. Now it is obvious that the family $\left(U_{i_{k}}^{*}\right)_{1 \leq k \leq n}$ is the family of open sets (in $\left.G_{f}\right)$ ) whose union also covers $G_{f}$.

Proof of the item (vi). Let $x^{*} \in G_{f}$. Since $(G, \odot, \rho)$ is locally compact the point $f^{-1}\left(x^{*}\right)$ has a compact neighbourhood $U$. Now it is obvious that the set $f(U)$ will be a compact neighbourhood of the point $x^{*}$. Since $x^{*} \in G_{f}$ was taken arbitrary the validity of the item (vi) is proved.

Proof of the item (vii). Since $(G, \odot, \rho)$ is no locally compact there is a point $x_{0}$ which has no a compact neighbourhood. Now if we consider a point $f\left(x_{0}\right)$, we observe that it has no a compact neighbourhood. Indeed, if assume the contrary and $U$ is a compact neighbourhood of the point $f\left(x_{0}\right)$ then $f^{-1}(U)$ also will be a compact neighbourhood of the point $x_{0}$ and we get the contradiction. This ends the proof of the item (vii).

Proof of the item (viii).

Proof of the diffusivity of the measure $\lambda_{f}$. Since $\lambda$ vanishes on all singletons, we have

$$
\left.\lambda_{f}(x)=\lambda\left(f^{-1}(x)\right)\right)=0
$$

for each $x \in G_{f}$;

Proof of the left(or right or two-sided) invariance of the measure $\lambda_{f}$. If $(G, \odot, \rho)$ is a locally compact or compact Polish group and $\lambda$ is a left(or right or two-sided ) invariant Haar measure in $(G, \odot, \rho)$, then $\lambda_{f}$ also will be a left(or right or two-sided ) invariant Haar measure in $\left(G_{f}, \odot_{f}, \rho_{f}, \mathcal{B}_{\rho_{f}}\left(G_{f}\right)\right)$, where $G_{f}=X, \mathcal{B}_{\rho_{f}}\left(G_{f}\right)$ is Borel $\sigma$-algebra of $G_{f}$ generated by the metric $\rho_{f}$ and $\lambda_{f}$ is defined by

$$
(\forall Y)\left(Y \in \mathcal{B}_{\rho_{f}}\left(G_{f}\right) \rightarrow \lambda_{f}(Y)=\lambda\left(f^{-1}(Y)\right)\right) .
$$


Case 1. $\lambda$ is a left invariant Haar measure in $(G, \odot, \rho)$.

$$
\begin{gathered}
(\forall Y)(\forall h)\left(\left(Y \in \mathcal{B}_{\rho_{f}}\left(G_{f}\right) \& h \in G_{f}\right) \rightarrow \lambda_{f}\left(h \odot_{f} Y\right)=\right. \\
\left.\left.\lambda\left(f^{-1}\left(h \odot_{f} Y\right)\right)=\lambda\left(f^{-1}(h) \odot f^{-1}(Y)\right)=\lambda\left(f^{-1}(Y)\right)=\lambda_{f}(Y)\right)\right) .
\end{gathered}
$$

Case 2. $\lambda$ is a right invariant Haar measure in $(G, \odot, \rho)$.

$$
\begin{gathered}
(\forall Y)(\forall h)\left(\left(Y \in \mathcal{B}_{\rho_{f}}\left(G_{f}\right) \& h \in G_{f}\right) \rightarrow \lambda_{f}\left(Y \odot_{f} h\right)=\right. \\
\left.\left.\lambda\left(f^{-1}\left(Y \odot_{f} h\right)\right)=\lambda\left(f^{-1}(Y) \odot f^{-1}(h)\right)=\lambda\left(f^{-1}(Y)\right)=\lambda_{f}(Y)\right)\right) .
\end{gathered}
$$

Case 3. $\lambda$ is a two-sided invariant Haar measure in $(G, \odot, \rho)$.

$$
\begin{gathered}
(\forall Y)\left(\forall h_{1}\right)\left(\forall h_{2}\right)\left(\left(Y \in \mathcal{B}_{\rho_{f}}\left(G_{f}\right) \& h_{1} \in G_{f}\right) \& h_{2} \in G_{f}\right) \rightarrow \lambda_{f}\left(h_{1} \odot_{f} Y \odot_{f} h_{2}\right)= \\
\left.\left.\lambda\left(f^{-1}\left(h_{1} \odot_{f} Y \odot_{f} h_{2}\right)\right)=\lambda\left(f^{-1}\left(h_{1}\right) \odot f^{-1}(Y) \odot f^{-1}\left(h_{2}\right)\right)=\lambda\left(f^{-1}(Y)\right)=\lambda_{f}(Y)\right)\right) .
\end{gathered}
$$

Proof of the outer regularity of the measure $\lambda_{f}$. Let take any set $E_{f} \in B_{\rho_{f}}\left(G_{f}\right)$ and any $\epsilon>0$. Let consider a set $f^{-1}\left(E_{f}\right) \in B(G)$. Since $\lambda$ is outer regular there is an open subset $U$ of $G$ such that $f^{-1}\left(E_{f}\right) \subseteq U$ and $\lambda\left(U \backslash f^{-1}\left(E_{f}\right)\right)<\epsilon$. Then we get

$$
\lambda_{f}\left(f(U) \backslash E_{f}\right)=\lambda\left(f^{-1}\left(f(U) \backslash E_{f}\right)\right)=\lambda\left(U \backslash f^{-1}\left(E_{f}\right)\right)<\epsilon .
$$

Proof of the inner regularity of the measure $\lambda_{f}$. Let take any set $E_{f} \in B_{\rho_{f}}\left(G_{f}\right)$ and any $\epsilon>0$. Let consider a set $f^{-1}\left(E_{f}\right) \in B(G)$. Since $\lambda$ is inner regular there is a compact subset $F$ of $G$ such that $F \subseteq f^{-1}\left(E_{f}\right)$ and $\lambda\left(f^{-1}\left(E_{f}\right) \backslash F\right)<\epsilon$. Then we get

$$
\lambda_{f}\left(E_{f} \backslash f(F)\right)=\lambda\left(f^{-1}\left(E_{f} \backslash f(F)\right)=\lambda\left(f^{-1}\left(E_{f}\right) \backslash F\right)<\epsilon .\right.
$$

Proof of the finiteness of the measure $\lambda_{f}$ on all compact subsets. Let take any compact set $F \subseteq G_{f}$. Since $f^{-1}(F)$ is compact in $G$ and the measure $\lambda$ is finite on every compact set we get $\lambda_{f}(F)=$ $\lambda\left(f^{-1}(F)\right)<\infty$.

Proof of the item (ix). The proof of this item can be obtained by the scheme used in the proof of the item (viii).

Below we consider some examples which employ the constructions described by Theorem 2.1.

Example 2.1 Let $f: R \rightarrow(-c, c)$ be defined by $f(y)=\frac{c\left(e^{y}-1\right)}{1+e^{y}}$ for $y \in R$, where $c>0$. Then $f^{-1}:(-c, c) \rightarrow R$ is defined by $f^{-1}(x)=\ln \left(\frac{c+x}{c-x}\right)$ for $x \in(-c, c)$. For $x, y \in(-c, c)$ we put

$$
\begin{aligned}
& x+{ }_{f} y=f\left(f^{-1}(x)+f^{-1}(y)\right)=f\left(\ln \left(\frac{c+x}{c-x}\right)+\ln \left(\frac{c+y}{c-y}\right)\right)=f\left(\ln \left(\frac{(c+x)(c+y)}{(c-x)(c-y)}\right)\right)= \\
& \frac{c\left(e^{\ln \left(\frac{(c+x)(c+y)}{(c-x)(c-y)}\right)}-1\right)}{1+e^{\ln \left(\frac{(c+x)(c+y)}{(c-x)(c-y)}\right)}}=\frac{c\left(\frac{(c+x)(c+y)}{(c-x)(c-y)}-1\right)}{1+\frac{(c+x)(c+y)}{(c-x)(c-y)}}= \\
& c \frac{(c+x)(c+y)-(c-x)(c-y)}{(c-x)(c-y)+(c+x)(c+y)}=c \frac{2 c x+2 c y}{2 c^{2}+2 x y}=\frac{x+y}{1+\frac{x y}{c^{2}}} .
\end{aligned}
$$


Note that $\lambda_{f}$ defined by

$$
(\forall Y)\left(Y \in \mathcal{B}_{\rho_{f}}((-c, c)) \rightarrow \lambda_{f}(Y)=\lambda\left(\left\{\ln \left(\frac{c+y}{c-y}\right): y \in Y\right\}\right)=\int_{Y} \frac{c^{2}}{c^{2}-t^{2}} d t\right)
$$

will be Haar measure in $(-c, c)$, where $\lambda$ denotes a linear Lebesgue measure in $R$.

Remark 2.1 Example 2.1 demonstrates that the Haar measure space $\left(G_{c}, \star, \rho_{G_{c}}, \nu\right)$ which comes from [4](cf. Example 9.1, p.61) exactly coincides with a Polish group $\left(R_{f},+_{f}, \rho_{f}, \lambda_{f}\right)$ where $\rho$ is a usual metric in $R, \lambda$ is a linear Lebesgue measure in $R$ and $f: R \rightarrow(-c, c)$ is a mapping defined by $f(y)=\frac{c\left(e^{y}-1\right)}{1+e^{y}}$ for $y \in R$. form

It is well known(see, [5], Eq. 35, p. 5) that the relativistic law of adding velocities has the following

$$
v=\frac{v_{1}+v_{2}}{1+\frac{v_{1} v_{2}}{c^{2}}}
$$

for $v_{1}, v_{2} \in(-c, c)$, where $c$ denotes the speed of light. This operation of adding exactly coincides with the operation $+_{f}$ under which $(-c, c)$ stands a locally compact non-compact Polish group. Hence the Haar measure $\lambda_{f}$ can be used in studding properties of the inertial reference frame $O_{0}$ which moves relative to $O$ with velocity $v$ in along the $x$ axis (see, [5], p. 1).

Example 2.2 Let $(R,+, \rho)$ be a one-dimensional Euclidian vector space and $\lambda$ a linear Lebesgue measure in $R$. Let $f: R \rightarrow(0,+\infty)$ be defined by $f(x)=e^{x}$. We put

$$
x+_{f} y=\exp \{\ln (x)+\ln (y)\}=\exp \{\ln (x y)\}=x y
$$

and

$$
\rho_{f}(x, y)=|\ln (x)-\ln (y)|
$$

for $x, y \in(0,+\infty)$. We define $\lambda_{f}$ by

$$
(\forall Y)\left(Y \in \mathcal{B}((0,+\infty)) \rightarrow \lambda_{f}(Y)=\lambda(\{\ln (y): y \in Y\})\right) .
$$

By Theorem 2.1 we know that $\lambda_{f}$ is Haar measure in $(0,+\infty)$. Since

$$
(\forall Z)\left(Z \in \mathcal{B}(R) \rightarrow \lambda(Z)=\int_{Z} d x\right)
$$

we deduce that

$$
(\forall Y)\left(Y \in \mathcal{B}((0,+\infty)) \rightarrow \lambda_{f}(Y)=\lambda(\ln (Y))=\int_{\ln (Y)} d x=\int_{Y} \frac{d x}{x}\right) .
$$

Note that Haar measure space $\left(G, \cdot, \rho_{G}, \nu\right)$ constructed in [4](see p.54) coincides with Haar measure space $\left(R_{f},+_{f}, \rho_{f}, \lambda_{f}\right)$.

Example 2.3 Let $X=(-c, c)$ where $c>0$. We define $f: R \rightarrow(-c, c)$ by $f(x)=\frac{2 \operatorname{carctg}(x)}{\pi}$ for $x \in R$. Then $f^{-1}(w)=\operatorname{tg}\left(\frac{\pi w}{2 c}\right)$ for $w \in(-c, c)$. We have

$$
x \odot_{f} y=f\left(f^{-1}(x) \odot f^{-1}(y)\right)=f\left(\operatorname{tg}\left(\frac{\pi x}{2 c}\right)+\operatorname{tg}\left(\frac{\pi y}{2 c}\right)\right)=\frac{2 \operatorname{carctg}\left(\operatorname{tg}\left(\frac{\pi x}{2 c}\right)+\operatorname{tg}\left(\frac{\pi y}{2 c}\right)\right)}{\pi}=
$$


and

$$
\rho_{f}(x, y)=\rho\left(f^{-1}(x), f^{-1}(y)\right)=\left|\operatorname{tg}\left(\frac{\pi x}{2 c}\right)-\operatorname{tg}\left(\frac{\pi y}{2 c}\right)\right|
$$

for $x, y \in(-c, c)$.

Then we get a new example of Haar measure space $\left(R_{f},{ }_{+}, \rho_{f}, \lambda_{f}\right)$. Note that the Haar measure $\lambda_{f}$ in $(-c, c)$ is defined by

$$
\left.(\forall Y)\left(Y \in \mathcal{B}_{f}((-c, c)) \rightarrow \lambda_{f}(Y)=\lambda\left(f^{-1}(Y)\right)\right)=\lambda\left(\left\{\operatorname{tg}\left(\frac{\pi w}{2 c}\right): w \in Y\right\}\right)\right) .
$$

Example 2.4 Let $f: R \rightarrow Z \times\{0,1, \cdots, 9\}^{N}$ be defined by $f\left(a_{0}+0, a_{1} a_{2} \cdots\right)=\left(a_{0}, a_{1}, a_{2}\right.$, $\cdots)$ for $a_{0} \in Z$ and $\left(a_{0}, a_{1}, a_{2}, \cdots\right) \in\{0,1, \cdots, 9\}^{N}$.

Then $f^{-1}: Z \times\{0,1, \cdots, 9\}^{N} \rightarrow R$ is defined by $f^{-1}\left(\left(a_{0}, a_{1}, a_{2}, \cdots\right)\right)=a_{0}+0, a_{1} a_{2} \cdots$. We put

$$
\begin{gathered}
\left(a_{0}, a_{1}, a_{2}, \cdots\right)+_{f}\left(b_{0}, b_{1}, b_{2}, \cdots\right)=f\left(f^{-1}\left(\left(a_{0}, a_{1}, a_{2}, \cdots\right)\right)+f^{-1}\left(\left(b_{0}, b_{1}, b_{2}, \cdots\right)\right)\right)= \\
f\left(a_{0}, a_{1} a_{2} \cdots+b_{0}, b_{1} b_{2} \cdots\right)=f\left(c_{0}, c_{1} c_{2} \cdots\right)=\left(c_{0}, c_{1}, c_{2} \cdots\right),
\end{gathered}
$$

where $c_{0}, c_{1} c_{2} \cdots=a_{0}, a_{1} a_{2} \cdots+b_{0}, b_{1} b_{2} \cdots$. The metric $\rho_{f}$ in $Z \times\{0,1, \cdots, 9\}^{N}$ is defined by

$$
\begin{gathered}
\rho_{f}\left(\left(a_{0}, a_{1}, a_{2}, \cdots\right),\left(b_{0}, b_{1}, b_{2}, \cdots\right)\right)=\rho\left(f^{-1}\left(\left(a_{0}, a_{1}, a_{2}, \cdots\right)\right), f^{-1}\left(\left(b_{0}, b_{1}, b_{2}, \cdots\right)\right)\right)= \\
\rho\left(a_{0}+0, a_{1} a_{2} \cdots, b_{0}+0, b_{1} b_{2} \cdots\right)=\left|\left(a_{0}+0, a_{1} a_{2} \cdots\right)-\left(b_{0}+0, b_{1} b_{2} \cdots\right)\right| .
\end{gathered}
$$

By Theorem 2.1 we know that $\lambda_{f}$ defined by

$$
\begin{gathered}
(\forall Y)\left(Y \in \mathcal{B}_{\rho_{f}}\left(Z \times\{0,1, \cdots, 9\}^{N}\right) \rightarrow \lambda_{f}(Y)=\lambda\left(f^{-1} Y\right)=\right. \\
\lambda\left(\left\{a_{0}, a_{1} a_{2} \cdots:\left(a_{0}, a_{1}, a_{2}, \cdots\right) \in Y\right)\right.
\end{gathered}
$$

is Haar measure in $Z \times\{0,1, \cdots, 9\}^{N}$, where $\lambda$ denotes a linear Lebesgue measure in $R$.

Remark 2.2 Let $M$ be a topological space.

A homeomorphism $\phi: U \rightarrow V$ of an open set $U \subseteq M$ onto an open set $V \subseteq R^{d}$ will be called a local coordinate chart (or just 'a chart') and $U$ is then a coordinate neighbourhood (or 'a coordinate patch') in $M$.

A $C^{\infty}$ differentiable structure, or smooth structure, on $M$ is a collection of coordinate charts $\phi_{\alpha}$ : $U_{\alpha} \rightarrow V_{\alpha} \subseteq R^{d}$ (same $d$ for all $\alpha$ 's) such that

(i) $M=\cup_{\alpha \in A} U_{\alpha}$;

(ii) any two charts are 'compatible': for every $\alpha, \beta$ the change of local coordinates $\phi_{\beta} \circ \phi_{\alpha}^{-1}$ is a smooth $C^{\infty}$ map on its domain of definition, i.e. on $\phi_{\alpha}\left(U_{\beta} \cap U_{\alpha}\right) \subseteq R^{d}$;

(iii) the collection of charts $\phi_{\alpha}$ is maximal with respect to the property (ii): if a chart $\phi$ of $M$ is compatible with all $\phi_{\alpha}$ then $\phi$ is included in the collection.

A topological space equipped with a $C^{\infty}$ differential structure is called a real smooth manifold. Then $d$ is called the dimension of $M, d=\operatorname{dim} M$.

Recall, that a Lie group is a set $G$ with two structures: $G$ is a group and $G$ is a real smooth manifold. These structures agree in the following sense: multiplication and inversion are smooth maps.

In [4](see, Example 9.7, p. 64), it is shown that $G=R^{k}$ with $k=\frac{n^{2}-n}{2}$ has two different Lie group structure and the Lebesgue measure in $R^{k}$ is Haar measure on both Lie groups. Further the author asks(cf. [4], Question 9.8) what are real $k$ - dimensional manifolds with at least two different Lie group structures that have the same Haar measure.

The next example answers positively to Maleki's above mentioned question for each $k>2$. 
Example 2.5 For $n>2$, let $\left(R^{n}, \rho_{n},+, \lambda_{n}\right)$ be an $n$-dimensional Euclidean vector space equipped with standard metric $\rho_{n}$ and $n$-dimensional Lebesgue measure $\lambda_{n}$. Let $f: R^{n} \rightarrow R^{n}$ be defined by $f\left(x_{1}, x_{2}, x_{3}, \cdots, x_{n}\right)=\left(x_{1}, x_{1}^{2}+x_{2}, x_{3}, \cdots, x_{n}\right)$ for $\left(x_{1}, x_{2}, x_{3}, \cdots, x_{n}\right) \in R^{n}$.

It is obvious that

1) $f$ is bijection of $R^{n}$ and

$$
f^{-1}\left(\left(x_{1}, x_{2}, x_{3}, \cdots, x_{n}\right)\right)=\left(x_{1}, x_{2}-x_{1}^{2}, x_{3}, \cdots, x_{n}\right)
$$

for $\left(x_{1}, x_{2}, x_{3}, \cdots, x_{n}\right) \in R^{n}$;

2) $f$ as well $f^{-1}$ is infinitely many times continuously differentiable;

3) $f$ is not linear;

4) $f$ as well $f^{-1}$ preserves Lebesgue measure $\lambda_{n}$.

By virtue of Theorem 2.1 we deduce that

$$
\left(\left(R^{n}\right)_{f},\left(\rho_{n}\right)_{f},+_{f},\left(\lambda_{n}\right)_{f}\right)
$$

is a locally compact non-compact Polish group with two-sided invariant Haar measure $\left(\lambda_{n}\right)_{f}$.

Note that $\left(R^{n}\right)_{f}=R^{n}$;

b) $\left(\rho_{n}\right)_{f}(x, y)=\rho_{n}\left(f^{-1}(x), f^{-1}(y)\right)$;

c) $x+{ }_{f} y=f\left(f^{-1}(x)+f^{-1}(y)\right)$

Note that the operation " ${ }_{f}^{\prime \prime}$ is commutative but it differs from the usual addition operation" ${ }^{\prime \prime}$. Indeed, we have

$$
\begin{gathered}
(1,1, \cdots, 1)+_{f}(2,2, \cdots, 2)=f\left(f^{-1}(1,1, \cdots, 1)+f^{-1}(2,2, \cdots, 2)\right)= \\
f((1,0,1, \cdots, 1)+(2,-2,2, \cdots, 2))=f(3,-2,3, \cdots, 3)=(3,7,3, \cdots, 3)
\end{gathered}
$$

and

$$
(1, \cdots, 1)+(2, \cdots, 2)=(3, \cdots, 3) .
$$

Since $f$ is Borel measurable, by using Theorem 2.1 we deduce that $\mathcal{B}_{\rho_{f}}\left(R^{n}\right)=\mathcal{B}\left(R^{n}\right)$. Note also that $\left(\lambda_{n}\right)_{f}=\lambda_{n}$. Indeed, by Theorem 2.1 we have that

$$
(\forall Y)\left(Y \in \mathcal{B}\left(R^{n}\right) \rightarrow \lambda_{f}(Y)=\lambda\left(f^{-1}(Y)\right)=\lambda(Y)\right) .
$$

Remark 2.3 Notice that Example 2.5 covers Example 9.7 from [4]. Indeed, it is obvious that for $n>2$, measure space $\left(\left(R^{n}\right)_{f},\left(\rho_{n}\right)_{f},+_{f},\left(\lambda_{n}\right)_{f}\right)=\left(R^{n},\left(\rho_{n}\right)_{f},+_{f}, \lambda_{n}\right)$ has Lie group structure which differs from standard Lie group structure of $R^{n}$ because group operations " $+^{\prime \prime}$ and ${ }^{\prime \prime}+{ }_{f}^{\prime \prime}$, as were showed in Example 2.5, are different. Furthermore the Lebesgue measure $\lambda_{n}$ (in $R^{n}$ ) is Haar measure on both Lie groups.

Now let us consider $\ell_{2}=\left\{\left(x_{k}\right)_{k \in N}: x_{k} \in R \& k \in N \& \sum_{k \in N} x_{k}^{2}<\infty\right\}$ as a vector space with usual addition operation " + ". If we equip $\ell_{2}$ with standard metric $\rho_{\ell_{2}}$ defined by

$$
\rho_{\ell_{2}}\left(\left(x_{k}\right)_{k \in N},\left(y_{k}\right)_{k \in N}\right)=\sqrt{\sum_{k \in N}\left(x_{k}-y_{k}\right)^{2}}
$$

for $\left(x_{k}\right)_{k \in N},\left(y_{k}\right)_{k \in N} \in \ell_{2}$, then $\left(\ell_{2},{ }^{\prime \prime}+{ }^{\prime \prime}, \rho_{\ell_{2}}\right)$ stands an example of a non-locally compact Polish group. Here naturally arise a question asking whether there exists a metric $\rho$ in $\ell_{2}$ such that $\left(\ell_{2}, "+,, \rho\right)$ stands an example of a locally compact $\sigma$-compact Polish group. An affirmative answer to this question is containing in the following example. 
Example 2.6 Let consider $R$ and $\ell_{2}$ as vector spaces over the group of all rational numbers $Q$. Let $\left(a_{i}\right)_{i \in I}$ and $\left(b_{i}\right)_{i \in I}$ be Hamel bases in $R$ and $\ell_{2}$, respectively. For $x \in R \backslash\{0\}$, there exists a unique sequence of non-zero rational numbers $\left(q_{i_{k}}^{(x)}\right)_{1 \leq k \leq n_{x}}$ such that $x=\sum_{k=1}^{n_{x}} q_{i_{k}}^{(x)} a_{i_{k}}$. We set $f(x)=\sum_{k=1}^{n_{x}} q_{i_{k}}^{(x)} b_{i_{k}}$ for $x \in R \backslash\{0\}$ and $f(0)=(0,0, \cdots)$. Note that $f: R \rightarrow \ell_{2}$ is one-to-one linear transformation.

Let $x=\sum_{k=1}^{n_{x}} q_{i_{k}}^{(x)} b_{i_{k}}$ and $y=\sum_{k=1}^{n_{y}} q_{i_{k}}^{(y)} b_{i_{k}}$. Now if we set

$$
x+{ }_{f} y=f\left(f^{-1}(x) \odot f^{-1}(y)\right),
$$

then we will obtain

$$
\begin{gathered}
x+_{f} y=f\left(f^{-1}(x)+f^{-1}(y)\right)=f\left(\sum_{k=1}^{n_{x}} q_{i_{k}}^{(x)} a_{i_{k}}+\sum_{k=1}^{n_{y}} q_{i_{k}}^{(y)} a_{i_{k}}\right)= \\
\sum_{k=1}^{n_{x}} q_{i_{k}}^{(x)} b_{i_{k}}+\sum_{k=1}^{n_{y}} q_{i_{k}}^{(y)} b_{i_{k}}=x+y,
\end{gathered}
$$

which means that a group operation $+_{f}$ coincides with usual addition operation " + ".

Let $\rho$ be defined by the formula

$$
\rho(x, y)=\left|f^{-1}(x)-f^{-1}(y)\right|=\left|\sum_{k=1} n_{x} q_{i_{k}}^{(x)} a_{i_{k}}-\sum_{k=1} n_{y} q_{i_{k}}^{(y)} a_{i_{k}}\right| .
$$

By Theorem 2.1 we know that $\left(R_{f},+_{f}, \rho_{f}\right)$, equivalently $\left(\ell_{2},+, \rho_{f}\right)$, is a locally compact noncompact Polish group which is isomorphic to the Polish group $(R,+,|\cdot|)$.

Moreover, if $(R,+,|\cdot|, \lambda)$ is Haar measure space, then $\left(\ell_{2},+, \rho_{f}, \lambda_{f}\right)$ also is Haar measure space. Denoting by $\mathcal{B}_{\rho_{f}}\left(\ell_{2}\right)$ a Borel $\sigma$-algebra of subsets of $\ell_{2}$ generated by the metric $\rho_{f}$, we define Haar measure $\lambda_{f}$ in $\ell_{2}$ by

$$
(\forall Y)\left(Y \in \mathcal{B}_{\rho_{f}}\left(\ell_{2}\right) \rightarrow \lambda_{f}(Y)=\lambda\left(f^{-1}(Y)\right)\right)
$$

Remark 2.4 Let $(G, \rho,+)$ be an abelian Polish group. We say that $G$ is one-dimensional group w.r.t. metric $\rho$ if for each $n \in N$ and for each family of different elements $\left(a_{k}\right)_{1 \leq k \leq n}$ there exists a permutation $h$ of $\{1,2, \cdots, n\}$ such that

$$
\rho\left(a_{h(1)}, a_{h(n)}\right)=\sum_{k=1}^{n-1} \rho\left(a_{h(k)}, a_{h(k+1)}\right) .
$$

Then it is obvious that $\left(\ell_{2},+, \rho_{f}, \lambda_{f}\right)$ is one-dimensional group w.r.t. metric $\rho_{f}$.

Example 2.7 Let consider $R^{\infty}$ and $R$ as vector spaces over the group of all rational numbers $Q$. Let $\left(a_{i}\right)_{i \in I}$ and $\left(b_{i}\right)_{i \in I}$ be Hamel bases in $R^{\infty}$ and $R$, respectively. For $x \in R^{\infty} \backslash\{(0,0, \cdots)\}$, there exists a unique sequence of non-zero rational numbers $\left(q_{i_{k}}^{(x)}\right)_{1 \leq k \leq n_{x}}$ such that $x=\sum_{k=1}^{n_{x}} q_{i_{k}}^{(x)} a_{i_{k}}$. We set $f(x)=\sum_{k=1}^{n_{x}} q_{i_{k}}^{(x)} b_{i_{k}}$ for $x \in R^{\infty} \backslash\{(0,0, \cdots)\}$ and $f(0,0, \cdots)=0$. Note that $f: R^{\infty} \rightarrow R$ is one-to-one linear transformation.

For $w, z \in R$, setting

$$
w+_{f} z=f\left(f^{-1}(w)+f^{-1}(z)\right)
$$


we get

$$
\begin{gathered}
w+{ }_{f} z=f\left(f^{-1}(w)+f^{-1}(z)\right)=f\left(\sum_{k=1}^{n_{w}} q_{i_{k}}^{(w)} a_{i_{k}}+\sum_{k=1}^{n_{z}} q_{i_{k}}^{(z)} a_{i_{k}}\right)= \\
\sum_{k=1}^{n_{w}} q_{i_{k}}^{(w)} b_{i_{k}}+\sum_{k=1}^{n_{z}} q_{i_{k}}^{(z)} b_{i_{k}}=w+z,
\end{gathered}
$$

which means that a group operation $+_{f}$ coincides with a usual addition operation " + " in $R$.

Let $\rho$ be defined by the formula

$$
\rho(w, z)=\rho_{T}\left(f^{-1}(w), f^{-1}(z)\right)
$$

where $\rho_{T}$ is Tychonov metric in $R^{\infty}$ defined by

$$
\rho_{T}\left(\left(x_{k}\right)_{k \in N},\left(y_{k}\right)_{k \in N}\right)=\sum_{k=1}^{\infty} \frac{\left|x_{k}-y_{k}\right|}{2^{k}\left(1+\left|x_{k}-y_{k}\right|\right)}
$$

for $\left(x_{k}\right)_{k \in N},\left(y_{k}\right)_{k \in N} \in R^{\infty}$.

By Theorem 2.1 we know that $\left(R_{f}^{\infty},+_{f}, \rho_{f}\right)$, equivalently, $\left(R,+, \rho_{f}\right)$ is an abelian non-locally compact Polish group which is isomorphic to the abelian non-locally compact Polish group $\left(R^{\infty},+, \rho_{T}\right)$.

Let $\lambda$ be a translation invariant quasifinite Borel measure in $R^{\infty}$ (see, for example, [1], [2]).

We put

$$
(\forall Y)\left(Y \in \mathcal{B}_{\rho_{f}}(R) \rightarrow \lambda_{f}(Y)=\lambda\left(f^{-1}(Y)\right)\right) .
$$

Since $\lambda$ is a quasifinite translation invariant Borel measure in $R^{\infty}$, by virtue of Theorem 2.1 we deduce that so is the measure $\lambda_{f}$ in $\left(R,+, \rho_{f}\right)$.

\section{Is an arbitrary diffused Borel probability measure in a Polish space Haar measure?}

The purpose of the present section is resolve Problem 1.4.

This useful ingredient for our investigation is given by the following lemma.

Lemma 3.1 ([3], Theorem 4.12, p.81) Let $E_{1}$ and $E_{2}$ be any two Polish topological spaces without isolated points. Let $\mu_{1}$ be a probability diffused Borel measure on $E_{1}$ and let $\mu_{2}$ be a probability diffused Borel measure on $E_{2}$. Then there exists a Borel isomorphism $\varphi:\left(E_{1}, B\left(E_{1}\right)\right) \rightarrow\left(E_{2}, B\left(E_{2}\right)\right)$ such that

$$
\mu_{1}(X)=\mu_{2}(\varphi(X))
$$

for every $X \in B\left(E_{1}\right)$.

The solution of the Problem 1.2 is contained in the following statement.

Theorem 3.1 Let $(G, \rho)$ be a Polish metric space which is dense-in-itself. Let $\mu$ be a diffused Borel probability measure defined in $(G, \rho)$. Then there exist a metric $\rho_{\varphi}$ and a group operation $\odot_{\varphi}$ in $G$ such that the following three conditions

(i) The class of Borel measurable subsets of $G$ generated by the metric $\rho_{\varphi}$ coincides with the class of Borel measurable subsets of the same space generated by the metric $\rho$,

(ii) $\left(G, \rho_{\varphi}, \odot_{\varphi}\right)$ is a compact Polish group

and

(iii) $\mu$ is a left (right or two-sided) invariant probability Haar measure in $\left(G, \rho_{\varphi}, \odot_{\varphi}\right)$

hold true. 
Proof. Let $\left(G_{2}, \rho_{2}, \odot_{2}\right)$ be a compact Polish group which is dense-in-itself equipped with two-sided invariant Haar measure $\lambda_{2}$. By Lemma 3.1, there exists a Borel isomorphism $\varphi:(G, B(G)) \rightarrow$ $\left(G_{2}, B\left(G_{2}\right)\right)$ such that

$$
\mu(X)=\lambda_{2}(\varphi(X))
$$

for every $X \in B(G)$.

We set

$$
x \odot_{\varphi} y=\varphi^{-1}\left(\varphi(x) \odot_{2} \varphi(y)\right)
$$

and

$$
\rho_{\varphi}(x, y)=\rho_{2}(\varphi(x), \varphi(y))
$$

for $x, y \in G$.

By Theorem 2.1 we know that $\left(G, \odot_{\varphi}, \rho_{\varphi}\right)$ is a compact Polish group without isolated points which is Borel isomorphic to the compact Polish group $\left(G_{2}, \odot_{2}, \rho_{2}\right)$ and a measure $\lambda_{\varphi}$, defined by

$$
(\forall Y)\left(Y \in \mathcal{B}\left(G_{2}\right) \rightarrow \lambda_{\varphi}(Y)=\lambda\left(\varphi^{-1}(Y)\right)\right),
$$

is a two-sided invariant Haar measure in $G$.

Since $\varphi:(G, B(G)) \rightarrow\left(G_{2}, B\left(G_{2}\right)\right)$ is Borel isomorphism, we deduce that

$$
\begin{gathered}
\left\{z: \rho_{\varphi}(x, z)<r\right\}=\left\{z: \rho_{2}(\varphi(x), \varphi(z))<r\right\}= \\
\varphi^{-1}\left(\left\{w: \rho_{2}(\varphi(x), w)<r\right\}\right) \in B\left(G_{2}\right)
\end{gathered}
$$

for each $x \in G$ and $r>0$.

Since $\mathcal{B}(G)$ is $\sigma$-algebra, we deduce that $\mathcal{B}_{\rho_{\varphi}}(G) \subseteq \mathcal{B}(G)$.

We have to show that $\mathcal{B}(G) \subseteq \mathcal{B}_{\rho_{\varphi}}(G)$. Assume the contrary and let $X \in \mathcal{B}(G) \backslash \mathcal{B}_{\rho_{\varphi}}(G)$. Since $\varphi:(G, \mathcal{B}(G)) \rightarrow\left(G_{2}, \mathcal{B}\left(G_{2}\right)\right)$ is Borel isomorphism, we deduce $\varphi(X) \in \mathcal{B}\left(G_{2}\right)$. Then, by Theorem 2.1 we deduce that $X \in B_{\rho_{\varphi}}(G)$ and we get the contradiction.

Remark 3.1 In the proof of Theorem 3.1, if under $\left(G_{2}, \rho_{2}, \odot_{2}\right)$ we take an abelian compact Polish group without isolated points and with a two-sided invariant Haar measure $\lambda$ then the group $\left(G, \rho_{\varphi}, \odot_{\varphi}\right)$ will be a compact abelian Polish group without isolated points. Similar phenomena is valid in the case when $\left(G_{2}, \rho_{2}, \odot_{2}\right)$ is a non-abelian compact Polish group without isolated points.

The solution of Problem 1.3 is contained in the following statement.

Theorem 3.2 Let $(G, \rho)$ be a Polish metric space which is dense-in-itself. Let $\mu$ be a diffused $\sigma$-finite non-finite Borel measure defined in $(G, \rho)$. Then there exist a metric $\rho_{\varphi}$, a group operation $\odot_{\varphi}$ in $G$ and the Borel measure $\mu^{\star}$ in $G$ such that the following conditions

(i) The class of Borel measurable subsets of $G$ generated by the metric $\rho_{\varphi}$ coincides with the class of Borel measurable subsets of the same space generated by the metric $\rho$,

(ii) $\left(G, \rho_{\varphi}, \odot_{\varphi}\right)$ is a non-compact locally compact Polish group,

(iii) The measures $\mu^{\star}$ and $\mu$ are equivalent,

and

(iv) $\mu^{\star}$ is a left (right or two-sided) invariant $\sigma$-finite non-finite Haar measure in $\left(G, \rho_{\varphi}, \odot_{\varphi}\right)$ hold true.

Proof. Let $\left(G_{2}, \rho_{2}, \odot_{2}\right)$ be a non-compact locally compact Polish group which is dense-in-itself with two-sided invariant $\sigma$-finite non-finite Haar measure $\lambda_{2}$ (for example, the real axis $\mathbf{R}$ with Lebesgue measure ). Let $\left(X_{k}^{(2)}\right)_{k \in N}$ be a partition of the $G_{2}$ into Borel measurable subsets such that $0<$ $\lambda_{2}\left(X_{k}^{(2)}\right)<+\infty$ for $k \in N$. 
We set

$$
\mu_{2}(X)=\sum_{k \in N} \frac{\lambda_{2}\left(X \cap X_{k}^{(2)}\right)}{2^{k} \lambda_{2}\left(X_{k}^{(2)}\right)}
$$

for $X \in \mathcal{B}\left(G_{2}\right)$.

Similarly, let $\left(Y_{k}\right)_{k \in N}$ be a partition of the $G$ into Borel measurable subsets such that $0<\mu\left(Y_{k}\right)<$ $+\infty$ for $k \in N$. We set

$$
\mu_{1}(Y)=\sum_{k \in N} \frac{\mu\left(Y \cap Y_{k}\right)}{2^{k} \mu\left(Y_{k}\right)}
$$

for $Y \in \mathcal{B}(G)$.

By Lemma 3.1, there exists a Borel isomorphism $\varphi:(G, B(G)) \rightarrow\left(G_{2}, B\left(G_{2}\right)\right)$ such that

$$
\mu_{1}(Y)=\mu_{2}(\varphi(Y))
$$

for every $Y \in B(G)$.

We set

$$
x \odot_{\varphi} y=\varphi^{-1}\left(\varphi(x) \odot_{2} \varphi(y)\right)
$$

and

$$
\rho_{\varphi}(x, y)=\rho_{2}(\varphi(x), \varphi(y))
$$

for $x, y \in G$.

By Theorem 2.1 we know that $\left(G, \odot_{\varphi}, \rho_{\varphi}\right)$ is a locally compact non-compact Polish group without isolated points which is Borel isomorphic to the non-compact locally compact Polish group $\left(G_{2}, \odot_{2}, \rho_{2}\right)$.

Now we put

$$
\mu^{\star}(X)=\sum_{k \in N} 2^{k} \lambda_{2}\left(X_{k}^{(2)}\right) \mu_{1}\left(X \cap \varphi^{-1}\left(X_{k}^{(2)}\right)\right)
$$

for $X \in \mathcal{B}(G)$.

We have to show that the measure $\mu^{\star}$ is a two-sided invariant measure in $G$. Indeed, for $h_{1}, h_{2} \in G$ and $X \in \mathcal{B}(G)$, we have

$$
\begin{gathered}
\mu^{\star}\left(h_{1} \odot_{\varphi} X \odot_{\varphi} h_{2}\right)=\sum_{k \in N} 2^{k} \lambda_{2}\left(X_{k}^{(2)}\right) \mu_{1}\left(\left(h_{1} \odot_{\varphi} X \odot_{\varphi} h_{2}\right) \cap \varphi^{-1}\left(X_{k}^{(2)}\right)\right)= \\
\sum_{k \in N} 2^{k} \lambda_{2}\left(X_{k}^{(2)}\right) \mu_{2}\left(\varphi\left[\left(h_{1} \odot_{\varphi} X \odot_{\varphi} h_{2}\right) \cap \varphi^{-1}\left(X_{k}^{(2)}\right]\right)=\right. \\
\sum_{k \in N} 2^{k} \lambda_{2}\left(X_{k}^{(2)}\right) \sum_{i \in N} \frac{\lambda_{2}\left(\varphi\left[\left(h_{1} \odot_{\varphi} X \odot_{\varphi} h_{2}\right) \cap \varphi^{-1}\left(X_{k}^{(2)}\right)\right] \cap X_{i}^{(2)}\right)}{2^{i} \lambda_{2}\left(X_{i}^{(2)}\right)}= \\
\sum_{k \in N} 2^{k} \lambda_{2}\left(X_{k}^{(2)}\right) \sum_{i \in N} \frac{\lambda_{2}\left(\varphi\left[\varphi^{-1}\left\{\varphi h_{1} \odot \varphi(X) \odot \varphi\left(h_{2}\right)\right\} \cap \varphi^{-1}\left(X_{k}^{(2)}\right)\right] \cap X_{i}^{(2)}\right)}{2^{i} \lambda_{2}\left(X_{i}^{(2)}\right)}= \\
\sum_{k \in N} 2^{k} \lambda_{2}\left(X_{k}^{(2)}\right) \sum_{i \in N} \frac{\left.\lambda_{2}\left(\left(\varphi h_{1} \odot \varphi(X) \odot \varphi\left(h_{2}\right)\right) \cap X_{k}^{(2)}\right) \cap X_{i}^{(2)}\right)}{2^{i} \lambda_{2}\left(X_{i}^{(2)}\right)}=
\end{gathered}
$$




$$
\begin{gathered}
\sum_{k \in N} \lambda_{2}\left(\left(\varphi h_{1} \odot \varphi(X) \odot \varphi\left(h_{2}\right)\right) \cap X_{k}^{(2)}\right)= \\
\lambda_{2}\left(\varphi h_{1} \odot \varphi(X) \odot \varphi\left(h_{2}\right)\right)=\lambda_{2}(\varphi(X))=\sum_{k \in N} 2^{k} \lambda_{2}\left(X_{k}^{(2)}\right) \mu_{2}\left(\varphi(X) \cap X_{k}^{(2)}\right)= \\
\sum_{k \in N} 2^{k} \lambda_{2}\left(X_{k}^{(2)}\right) \mu_{1}\left(\varphi^{-1}\left[\varphi(X) \cap X_{k}^{(2)}\right]\right)= \\
\sum_{k \in N} 2^{k} \lambda_{2}\left(X_{k}^{(2)}\right) \mu_{1}\left(X \cap \varphi^{-1}\left(X_{k}^{(2)}\right)\right)=\mu^{\star}(X) .
\end{gathered}
$$

Remark 3.1 The result of Theorem 3.2 remains true if $\mu$ is a diffused Borel probability measure in $(G, \rho)$.

As a simple consequence of Theorem 3.2, we have the following corollary.

Corollary 3.1 Let $(G, \rho)$ be a Polish metric space which is dense-in-itself. Let $\mu$ be a diffused $\sigma$ finite non-finite Borel measure defined in $(G, \rho)$. Then there exist a metric $\rho_{\varphi}$ and a group operation $\odot_{\varphi}$ in $G$ such that the following three conditions

(i) The class of Borel measurable subsets of $G$ generated by the metric $\rho_{\varphi}$ coincides with the class of Borel measurable subsets of the same space generated by the metric $\rho$,

(ii) $\left(G, \rho_{\varphi}, \odot_{\varphi}\right)$ is a non-compact locally compact Polish group

and

(iii) The measure $\mu$ is a two-sided quasi-invariant ${ }^{4}$ Borel probability measure in $\left(G, \rho_{\varphi}, \odot_{\varphi}\right)$

hold true.

Finally, we state the following problem

Problem 3.1 Let $(G, \rho)$ be a Polish metric space which is dense-in-itself. Let $\mu$ be a diffused non-finite $\sigma$-finite Borel measure defined in $(G, \rho)$. Do there exist a metric $\rho_{1}$ and a group operation $\odot$ in $G$ such that the following three conditions

(j) The class of Borel measurable subsets of $G$ generated by the metric $\rho_{1}$ coincides with the class of Borel measurable subsets of the same space generated by the metric $\rho$,

(jj) $\left(G, \rho_{1}, \odot\right)$ is a non-compact locally compact Polish group

and

(jjj) $\mu$ is a left(right or two-sided) invariant non-finite $\sigma$-finite Haar measure in $\left(G, \rho_{1}, \odot\right)$ hold true?

Acknowledgement. The main results of this manuscript were reported on Swedish-Georgian Conference in Analysis \& Dynamical Systems which was held at National Academy of Georgian Republic in Tbilisi, Georgia, 15-22 July, 2015.

The designated project has been fulfilled by financial support of Shota Rustaveli National Science Foundation ( Grant: \# GNFS / FR 1165-100 / 14)

\footnotetext{
${ }^{4}$ A Borel measure $\mu$ defined in a Polish group $(G, \odot, \rho)$ is called two-sided quasi-invariant measure in $G$ if for each Borel subset $X$ we have $\mu(X)>0$ if and only $\mu\left(h_{1} \odot X \odot h_{2}\right)>0$ for each pair of elements $h_{1}, h_{2} \in G$.
} 


\section{References}

[1] R. Baker, "Lebesgue measure" on $R^{\infty}$. Proc. Amer. Math. Soc., 113(4) (1991), 1023-1029.

[2] R. Baker, "Lebesgue measure" on $\mathbb{R}^{\infty}$. II. Proc. Amer. Math. Soc., 132(9) (2004), 2577-2591.

[3] J. Cichon, A. Kharazishvili, Weglorz B., Subsets of the real line, 177 p. Wydawnictwo Uniwersytetu Lodzkiego, Lodz, 1995.

[4] A. Maleki, An applications of ultrafilters to the Haar measure, Afr. Diaspora J. Math., 14(1) (2012), 54-64.

[5] V. Yakovenko, Derivation of the Lorentz Transformation, Lecture note for course Phys171H, Introductory Physics: Mechanics and Relativity, Department of Physics, University of Maryland, College Park, 15 November, (2004), 1-5 .

http://www2.physics.umd.edu/ yakovenk/teaching/Lorentz.pdf

[6] R.P. Halmos, Measure Theory, xi+304 pp. D. Van Nostrand Company, Inc., New York, 1950.

[7] J. von Neumann, Invariant measures, xvi+134 pp. Amer. Math. Soc., Providence, RI, 1999.

[8] A. Haar, "Der Massbegriff in der Theorie der kontinuierlichen Gruppen", Ann. of Math., (2) 34 (1) (1933), 147-169. 